\title{
A Systematic Review of the Treatment of Fears and Phobias Among Children with Autism Spectrum Disorders
}

\author{
Sinéad Lydon • Olive Healy • Orla O'Callaghan • \\ Teresa Mulhern • Jennifer Holloway
}

Received: 24 June 2014 / Accepted: 21 November 2014 / Published online: 2 December 2014

(C) Springer Science+Business Media New York 2014

\begin{abstract}
Research indicates that fears and phobias are significantly more prevalent and emerge in response to a greater variety of stimuli, among children with autism spectrum disorders (ASD) than among their developmentally disabled or typically developing peers. Such findings are problematic given the difficulty of assessing and identifying fears or phobias among the ASD population and the challenge of identifying effective treatments for those with core diagnostic deficits in comprehension, communication, and attentional skills. The current review aimed to evaluate the literature describing interventions to treat fears, specific phobia, or social phobia among children with ASD and to identify evidence-based practice in this area. The review indicated that a variety of interventions, described as both traditional and novel, were successful in treating fearful or phobic behavioral responses to stimuli. The findings also suggest that behavioral intervention, including reinforcement, modeling, and exposure, may be considered evidence-based practice in the treatment of fears and phobias among children with ASD. However, the current research base is limited by the predominant focus on the behavioral element of the fear response, and the lack of research examining the cognitive or physiological responses during assessment or treatment.
\end{abstract}

Keywords Fear $\cdot$ Phobia $\cdot$ ASD $\cdot$ Autism $\cdot$ Asperger . Intervention $\cdot$ Treatment

This research was supported by the Irish Research Council's EMBARK Postgraduate Scholarship Scheme [RS/2012/134]

S. Lydon $\cdot$ O. Healy $(\bowtie)$

School of Psychology, Trinity College Dublin, College Green,

Dublin 2, Ireland

e-mail: olive.healy@tcd.ie

O. O'Callaghan · T. Mulhern · J. Holloway

National University of Ireland, Galway, Ireland
Fears are unpleasant feelings arising in response to a real or perceived threat (Marks 1987) while a phobia can be understood as an official diagnosis of a fear, which may be more severe, long-lasting, or age-inappropriate in nature (Turner and Romanczyk 2012). Fears are considered to be part of normal development; however, they can become maladaptive and problematic if they do not continue to progress in a normative fashion (Evans et al. 2005). Phobias, both social and specific, are categorized as anxiety disorders in the Diagnostic and Statistical Manual of Mental Disorders (5th ed.; DSM-5; American Psychiatric Association [APA] 2013). A fear evolves into a phobia when an individual's response to a stimulus or event becomes increasingly extreme or irrational in nature (APA 2013).

Social phobia is defined as marked and persistent fear, of one or more social or performance situations, in which the person is exposed to unfamiliar people or to possible scrutiny by others; the individual fears that he or she will act in a way that will be humiliating or embarrassing. Specific phobia is described as a marked and persistent fear that is excessive or unreasonable, cued by the presence or anticipation of a specific object or situation. Research indicates that the lifetime prevalence of specific phobias is over $10 \%$ making it one of the most prevalent and chronic psychological disorders (LeBeau et al. 2010; Van Houtem et al. 2013).

For both conditions, the phobic situation is either avoided or endured with a great deal of anxiety or distress (APA 2013). Lang (1968) states that the response to a feared stimulus is multifaceted as it has cognitive, physiological, and behavioral dimensions. The cognitive reactions may include false perceptions of the situation such as the belief that the stimulus or event poses a greater threat to the individual than it does in reality. Behavioral responses may include wincing, crying, shaking, and the emergence of avoidance behaviors such as physically attempting to escape from the situation or stimulus. Finally, the physiological impact of a feared stimulus may 
include activation of the autonomic nervous system (i.e., increased heart rate, blood pressure, and skin conductance) and the limbic-hypothalamic-pituitary-adrenal axis (i.e., release of the stress hormone, cortisol; Nesse et al. 1985). All three response components are suggested to occur simultaneously in the presence of the feared stimulus (Lang 1968).

Research indicates that fears and phobias are significantly more prevalent among individuals with autism spectrum disorders (ASD) than among their typically developing peers (Leyfer et al. 2006). The prevalence of specific phobia within the typically developing population is between 5 and $18 \%$ (Lichtenstein and Annas 2000; Muris et al. 2000; Ollendick et al. 2002), however, this figure is estimated to be significantly higher within the ASD population, with several studies reporting prevalence rates of between 30 and $64 \%$ (Leyfer et al. 2006; Muris et al. 1998; Van Steensel et al. 2011). Similarly, the incidence of social phobia in typically developing children is reported to be between 1 and $11 \%$ (Beesdo et al. 2009) and between 7.5 and $20.5 \%$ for children with ASD (Leyfer et al. 2006; Muris et al. 1998; Van Steensel et al. 2011).

The types of fears and phobias with which children present also vary significantly between those with and without ASD, with the type of fears in the ASD group often found to be more "unusual." Common childhood fears include fears of animals, death or injury, the dark, thunderstorms, and monsters or ghosts (Mayes et al. 2013), and although children with ASD often display these fears, they may also demonstrate fearful reactions towards what may be considered more unusual or atypical stimuli. Research suggests that less than $5 \%$ of those without ASD demonstrate unusual fears (Mayes et al. 2013) while up to $40 \%$ of those with ASD present with fears, which can be described as unusual, atypical, or uncommon (Mayes et al. 2013; Mayes et al. 2012). Turner and Romanczyk (2012) have described these rates to be as high as $64 \%$ but somewhat masked due to the difficulty of assessing fears and phobias among those with ASD. Unusual fears described in the literature have included fears of specific situations, noises, other people, toilets, shadows, clouds in the sky, and mechanical objects (Evans et al. 2005; Matson and Love 1990; Mayes et al. 2013). Evans et al. (2005) compared the fears and phobias of children with ASD and Down syndrome. Their findings indicated that children with ASD presented with more situation phobias and medical fears than typically developing children and children with Down syndrome. Furthermore, among those with ASD, a greater correlation between fears, phobias, and challenging behaviors was identified. To date, the source of such fears and the reason for the higher prevalence of unusual fears in children with ASD in comparison to typically developing children remains one of the many issues to be examined in ASD assessment and intervention research (Mayes et al. 2013).

The difficulty in assessing anxiety and phobias among those with ASD has been noted as a significant impediment to research and treatment in this area (Turner and Romanczyk
2012). The challenge of distinguishing between an anxious or fearful response and the symptoms of ASD, or the atypical or challenging behaviors often associated with the disorder, may lead to a failure to recognize or differentiate phobias or fears among children with ASD (Grondhuis and Aman 2012; Tsai 2006; Turner and Romanczyk 2012). Grondhuis and Aman (2012) note that clinicians are encouraged not to diagnose cooccurring disorders of which the symptoms may overlap with the primary diagnosis of autism; clinicians, therefore, often attribute the problems arising from the co-occurring condition or problems associated with fears or phobias to the symptoms of the primary diagnosis. Furthermore, children with ASD may have difficulties identifying, labeling, and reporting the emotions or feelings that they experience, a skill required for the completion of self-report measures or clinician interaction typically included in diagnostic assessments, given the language and cognitive deficits often associated with the disorder (Grondhuis and Aman 2012; Rieffe et al. 2007; Turner and Romanczyk 2012). Even among high-functioning individuals with ASD who do not present with such deficits, the assessment of anxious feelings may not be straightforward; White et al. (2012) found that a group of adolescents with highfunctioning ASD who were undergoing treatment for anxiety problems underreported their anxiety as compared to parent and clinician reports. There is also a noted lack of reliable and valid instruments for the diagnosis of phobias and fears among individuals with autism, particularly, those who are cognitively lower-functioning, which likely contributes to the underrecognition and under-diagnosis of these problems among children with autism (Reaven 2009; Turner and Romanczyk 2012); Tsai 2006). Such difficulties have likely impacted significantly upon both research and treatment in the area of fears and phobias in children with ASD.

In the typically developing population, an array of treatment approaches has been evaluated to treat fears or phobias. Reviews of the extant interventional research suggest that the primary treatment methods include systematic desensitization, reinforced practice, modeling, cognitive-behavioral therapy, and contingency management (Davis and Ollendick 2005; Ollendick and King 1998). A review conducted by Davis and Ollendick (2005) indicated that reinforced practice and modeling showed the greatest degree of empirical support for their efficacy in reducing fearful or phobic reactions to stimuli. Other methods including systematic desensitization and cognitive behavioral therapy could also be classified as likely efficacious based on current research support. However, these methods to date all focus on the cognitive and/or behavioral elements of the fear response. Davis and Ollendick (2005) highlight this as a limitation of the current body of research as the physiological component of the fear response has often been ignored or not considered in the treatment literature. Jennett and Hagopian (2008) reviewed the interventions used to treat phobias for persons with 
intellectual disabilities and categorized the intervention approaches employed as in vivo exposure, use of a fear hierarchy, contingent reinforcement, prompting procedures, modeling, extinction or response blocking, and the use of distracting stimuli. The authors highlighted that the behavioral treatment of phobias among those with intellectual disabilities emerged as evidence-based practice. Jennett and Hagopian (2008) also noted the widespread use of the avoidance behavior as the dependent variable for these studies, and a limited focus on the physiological component of the fear response, similar to that noted by Davis and Ollendick (2005) in the typically developing treatment literature.

Currently, there has been no attempt to explore or synthesize the literature examining interventions for fears and phobias for those on the autism spectrum. Such a review is warranted for several reasons. First, the high prevalence of fears and phobias among individuals diagnosed with ASD reported in the literature (e.g., Leyfer et al. 2006; Muris et al. 1998; Van Steensel et al. 2011) suggests that practitioners may frequently encounter clients for whom fears and phobias are negatively impacting daily functioning and which must be treated. In such cases, recommendations for best practice with regards to treatment would be invaluable. Second, the treatment responsivity of children with ASD may differ from those of the typically developing population, or of children with other developmental disabilities, and specific treatment approaches or interventions that have demonstrated efficacy with these individuals may be ineffective or counterproductive if employed with those diagnosed with ASD. For example, persons with ASD may show specific deficits in areas such as communication/language, visualization skills, the ability to attend and to remain on-task, comprehension skills, and imitation skills, required for the interventions most commonly applied to treat fears and phobias including modeling, cognitive behavioral therapy, and systematic desensitization (Ellis et al. 2006). In addition to this, previous research has suggested a correlation between the presence of fears and phobias and engagement in challenging behavior among children with ASD (Evans et al. 2005). It is possible that, for some individuals with ASD, challenging behavior may be a maladaptive response to feared or phobic stimuli. Given the negative impact of challenging behavior upon quality of life (Emerson 2001), it is important to examine whether fears or phobias can contribute to engagement in such behaviors and whether treatment intended to reduce the fear or phobia results in corresponding decreases in challenging behavior. An examination of extent research that attempts to assess and characterize the association between challenging behavior and fears or phobias may provide useful suggestions for the assessment and treatment of challenging behaviors among children with autism.

The current paper sought to evaluate the extant research examining interventions for fears and/or phobias in children with ASD. It was intended that this systematic literature review would provide a useful overview of the types of interventions being used to treat fears or phobias among children with ASD and the efficacy of these treatments for reducing the fear response. This paper also aimed to examine whether the three components of the fear response (i.e., cognitive, behavioral, and physiological elements) were targeted in the extant research, as the non-consideration of each of these components has been identified as a weakness of the treatment literature for typically developing persons and persons with intellectual disabilities. Given the difficulties of assessing fears and phobias among children with ASD (e.g., Grondhuis and Aman 2012; Reaven 2009; Tsai 2006), the measurement of the physiological component of fears or phobias may offer one means of gaining a better insight into the child's experience and providing more effective intervention.

\section{Method}

\section{Literature Search}

Articles were identified for inclusion by conducting comprehensive searches of six electronic databases: Scopus, PsycInfo, Web of Science, ScienceDirect, ERIC, and Psychology \& Behavioral Sciences Collection. Searches were carried out by inputting either fear or phobia in combination with autis*, ASD, asperger, pervasive developmental disorder, or developmental disability. In addition to these electronic searches, a subsequent review of the reference lists of all included studies was conducted by hand in order to identify any other suitable studies for inclusion.

\section{Inclusion Criteria}

The criteria for inclusion in the review were: (a) the application of an intervention to treat a fear, specific phobia, or social phobia; (b) the inclusion of participant(s) with a diagnosis of ASD; (c) the inclusion of participants below the age of 18 years; and (d) publication in an English language, peerreviewed journal.

\section{Evidence-Based Practice}

Chambless and Hollon's (1998) criteria for establishing the empirical support for a treatment or therapy, as applied to a particular problem or population, were used to determine the empirical support for interventions implemented to treat fears and phobias among those with ASD. These criteria suggest that for an intervention to be classified as evidence-based there must be at least two, well-designed, independently conducted, between-group experiments which have indicated that it is 
effective, or if three or more independently conducted, methodologically rigorous, single-case experimental designs have demonstrated positive outcomes with at least nine participants. A treatment may be considered promising but lacking sufficient evidence if initial research results demonstrate positive outcomes but the number of studies conducted is less than that required for the establishment of evidence-based practice or if the same team of researchers has conducted the studies evidencing positive outcomes. A treatment may be considered ineffective if there are three or more studies demonstrating poor outcomes with at least nine participants.

To ensure the accuracy of this analysis, a second coder reviewed all included studies and completed the evidencebased practice analysis independently. Agreement between the raters was subsequently assessed across both the summary of research support for each intervention employed (i.e., number of participants in randomized controlled trials with positive outcomes, number of participants in groups designs with positive outcomes, number of participants in single-subject research designs with positive outcomes, number of participants in case studies with positive outcomes) and the categorization of the level of evidence (i.e., evidence-based, promising but lacking sufficient evidence, or ineffective). In this way, there were two chances for raters to agree for each intervention. Interobserver agreement was found to be $96.6 \%$.

\section{Results}

A total of 16 studies met the inclusion criteria. Table 1 summarizes sample characteristics, the fears or phobias targeted, assessment of the fear or phobia employed, experimental design used, interventions implemented, and intervention outcomes.

\section{Participants}

With the exception of two studies (Gillis et al. 2009; Isong et al. 2014), all other studies had a sample size of three or fewer participants. A total of 117 participants diagnosed with an ASD received intervention across the studies reviewed. The majority $(82.9 \%)$ of these participates were male $(n=$ 97). Participants' age ranged from 2.5 to 18 years with a mean age of 9.2 years. Almost all $(96.6 \%)$ participants had a diagnosis of Autism, while the remaining participants had a diagnosis of either Asperger Syndrome $(n=2$; Karnezi and Tierney 2009; Schleismann and Gillis 2011) or Pervasive Developmental Disorder ( $n=2$; Davis et al. 2007; Gillis et al. 2009). Across the studies, three participants had a cooccurring diagnosis of intellectual disability.
Types of Fears or Phobias

There were a variety of stimuli identified as the source of the fear or phobia in the studies. As outlined in Table 1, these stimuli included fears of dogs, water, heights, skin lotions, doctors, auditory stimuli, going outside, the shower, dentists, swimming pools, animatronic objects, particular locations and activities, social situations, needle phobia, and riding the school bus.

Association between Fears or Phobias and Challenging Behavior

Included studies were assessed to determine whether challenging behaviors were reported to occur in the presence of the targeted fear or phobia. Twelve of the 16 studies reviewed (Chok et al. 2010; Davis et al. 2007; Ellis et al. 2006; Gillis et al. 2009; Jackson and King 1982; Koegel et al. 2004; Love et al. 1990; Luiselli 1978; Rapp et al. 2005; Ricciardi et al. 2006; Schmidt et al. 2013; Shabani and Fisher 2006) reported the occurrence of challenging behavior in the presence of the feared stimulus prior to intervention. Most common were disruptive vocalizations and verbalizations, such as screaming or crying, which were elicited in the presence of the feared or phobic stimulus in 11 studies (Chok et al. 2010; Ellis et al. 2006; Gillis et al. 2009; Jackson and King 1982; Koegel et al. 2004; Love et al. 1990; Luscre and Center 1996; Rapp et al. 2005; Ricciardi et al. 2006; Schmidt et al. 2013; Shabani and Fisher 2006). Elopement, or attempts to elope, was described by 10 studies (Chok et al. 2010; Gillis et al. 2009; Jackson and King 1982; Koegel et al. 2004; Love et al. 1990; Luiselli 1978; Rapp et al. 2005; Ricciardi et al. 2006; Schmidt et al. 2013; Shabani and Fisher 2006). Topographies of self-injury such as hitting the face or head, self-biting, and self-choking were reported by five of the studies reviewed (Chok et al. 2010; Davis et al. 2007; Rapp et al. 2005; Schmidt et al. 2013; Shabani and Fisher 2006), while topographies of aggressive behavior were reported by a further five studies (Davis et al. 2007; Ellis et al. 2006; Ricciardi et al. 2006; Schmidt et al. 2013; Shabani and Fisher 2006). Other challenging behaviors reportedly elicited by fearful or phobic stimuli included property destruction or misuse (Gillis et al. 2009; Schmidt et al. 2013) and tantrum behaviors (Davis et al. 2007; Jackson and King 1982; Luiselli 1978).

\section{Measurement of the Fear or Phobia}

The primary means of assessment of the targeted fear or phobia were the measurement of behavioral frequency $(n=$ 15; Davis et al. 2007; Ellis et al. 2006; Gillis et al. 2009; Isong et al. 2014; Jackson and King 1982; Karnezi and Tierney 2009; Koegel et al. 2004; Love et al. 1990; Luiselli 1978; Luscre and Center 1996; Rapp et al. 2005; Ricciardi et al. 


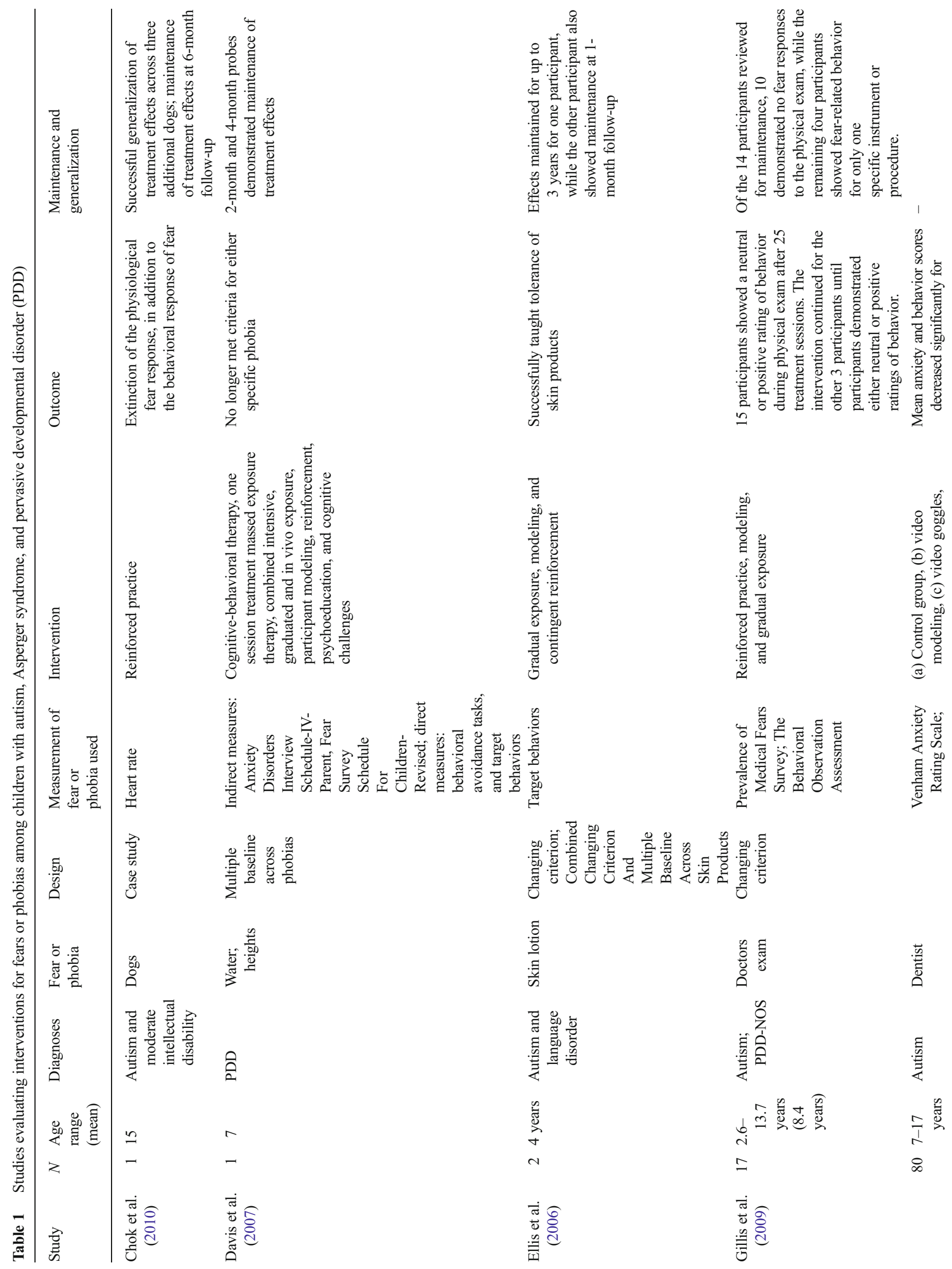




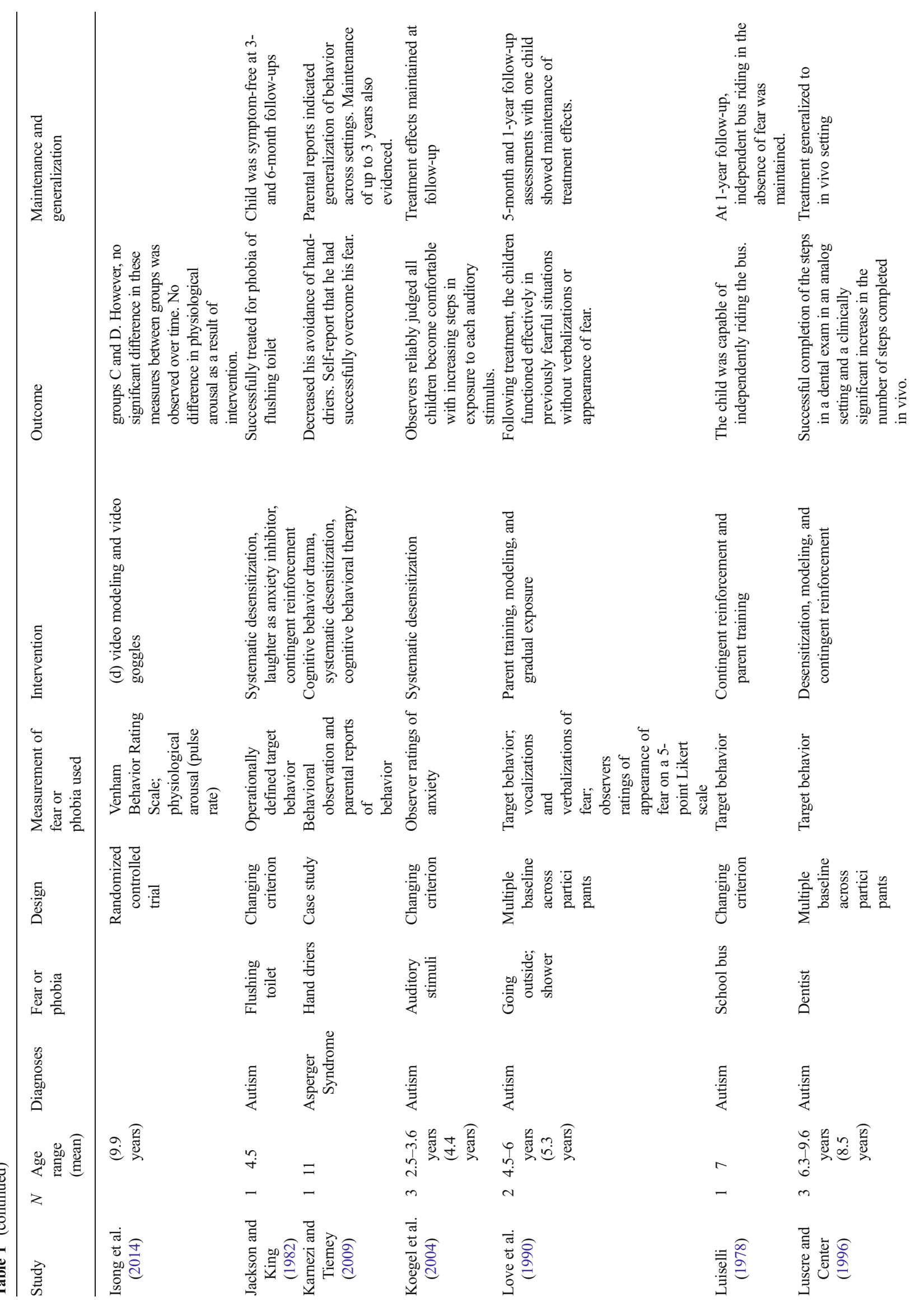




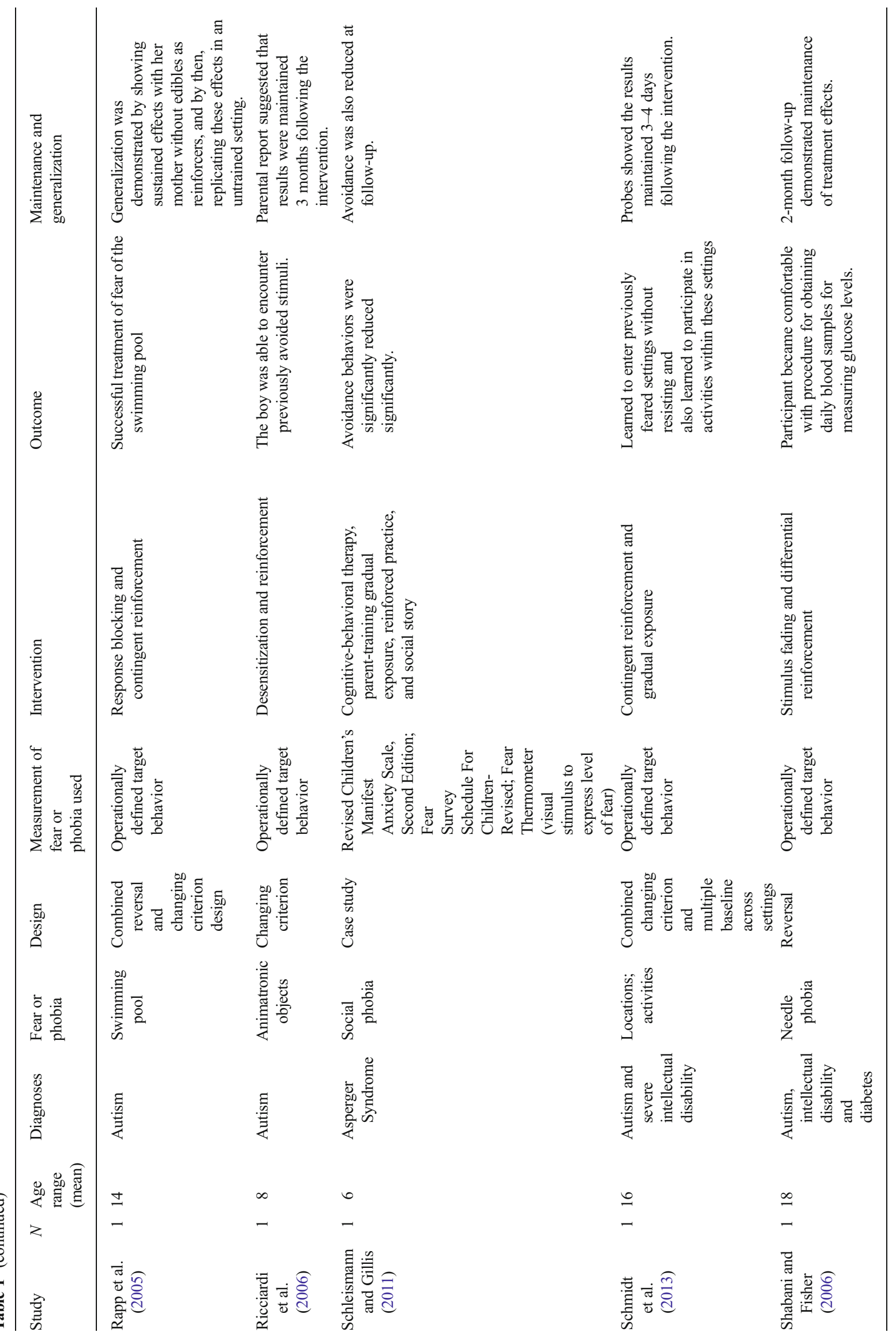


2006; Schmidt et al. 2013; Shabani and Fisher 2006). The behaviors measured were labeled by the researchers as avoidant and/or escape and/or fearful $(n=10$; Davis et al. 2007; Ellis et al. 2006; Gillis et al. 2009; Karnezi and Tierney 2009; Koegel et al. 2004; Love et al. 1990; Luscre and Center 1996; Ricciardi et al. 2006; Schleismann and Gillis 2011; Schmidt et al. 2013). These definitions included behavioral avoidance, attempts to escape the presence of the feared stimulus when presented, attempts to push or throw the feared stimulus, and overt distress including screaming and crying in the presence of the stimulus. Behaviors labeled as approach/ acceptance responses $(n=6$; Davis et al. 2007; Ellis et al. 2006; Gillis et al. 2009; Love et al. 1990; Ricciardi et al. 2006; Shabani and Fisher 2006) included compliant participation, engagement, or interaction with the feared stimulus or approach steps towards the target stimulus. In addition to this, several studies included the measurement of other behavioral responses, such as distance from the feared stimulus $(n=8$; Davis et al. 2007; Ellis et al. 2006; Koegel et al. 2004; Love et al. 1990; Luscre and Center 1996; Ricciardi et al. 2006; Schmidt et al. 2013; Shabani and Fisher 2006) and verbalizations of fear ( $n=1$; Love et al. 1990). Two studies employed a self-report measure of fear (Davis et al. 2007; Schleismann and Gillis 2011). A further two studies (Chok et al. 2010; Isong et al. 2014) utilized physiological measurements, including the measurement of heart and pulse rate responses as an indicator of fear. Less than half of the studies employed more than one measurement of fear or phobias $(n=$ 6; Davis et al. 2007; Gillis et al. 2009; Isong et al. 2014; Karnezi and Tierney 2009; Love et al. 1990; Schleismann and Gillis 2011), with one study utilizing a combination of physiological and behavioral measurements (Isong et al. 2014).

In sum, of the studies included, fifteen studies utilized a behavioral measurement of fear or phobias, two employed physiological measurements, while two studies administered measures of cognitive responses pre-intervention which were completed by the participating child, with varying degrees of assistance (Davis et al. 2007; Schleismann and Gillis 2011).

\section{Experimental Design}

Table 1 shows that the majority of studies employed singlesubject research designs $(n=15)$ with the exception of one randomized controlled trial (Isong et al. 2014). The singlesubject research designs within this review included a changing criterion design ( $n=8$; Ellis et al. 2006; Gillis et al. 2009; Jackson and King 1982; Koegel et al. 2004; Luiselli 1978; Rapp et al. 2005; Ricciardi et al. 2006; Schmidt et al. 2013), a multiple baseline design ( $n=7$; Davis et al. 2007; Ellis et al. 2006; Love et al. 1990; Luscre and Center 1996; Rapp et al. 2005; Ricciardi et al. 2006; Schmidt et al. 2013), a case study $(n=3$; Chok et al. 2010; Karnezi and Tierney 2009;
Schleismann and Gillis 2011), and/or a reversal design ( $n=$ 2; Rapp et al. 2005; Shabani and Fisher 2006). Two studies used a combination of changing criterion and multiple baseline research designs (Ellis et al. 2006; Schmidt et al. 2013), and one study incorporated both changing criterion and reversal designs (Rapp et al. 2005).

\section{Types of Interventions}

The studies reviewed used a variety of interventions to treat fears or phobias among those with ASD. These included (gradual) exposure ( $n=6$; Davis et al. 2007; Ellis et al. 2006; Gillis et al. 2009; Love et al. 1990; Schleismann and Gillis 2011; Schmidt et al. 2013), contingent reinforcement ( $n=11$; Chok et al. 2010; Ellis et al. 2006; Gillis et al. 2009; Jackson and King 1982; Luiselli 1978; Luscre and Center 1996; Rapp et al. 2005; Ricciardi et al. 2006; Schleismann and Gillis 2011; Schmidt et al. 2013; Shabani and Fisher 2006), modeling ( $n=$ 5; Davis et al. 2007; Ellis et al. 2006; Gillis et al. 2009; Love et al. 1990; Luscre and Center 1996), reinforced practice ( $n=$ 3; Chok et al. 2010; Gillis et al. 2009; Schleismann and Gillis 2011), systematic desensitization ( $n=5$; Jackson and King 1982; Karnezi and Tierney 2009; Koegel et al. 2004; Luscre and Center 1996; Ricciardi et al. 2006), parent training ( $n=2$; Love et al. 1990; Schleismann and Gillis 2011), cognitivebehavioral therapy $(n=3$; Davis et al. 2007; Karnezi and Tierney 2009; Schleismann and Gillis 2011), stimulus fading $(n=1$; Shabani and Fisher 2006), social stories $(n=1$; Schleismann and Gillis 2011), and response blocking $(n=1)$. In addition to the aforementioned interventions, the use of less common interventions, such as laughter as an anxiety inhibitor ( $n=1$; Jackson and King 1982), video goggles ( $n=1$; Isong et al. 2014), and cognitive behavior drama ( $n=1$; Karnezi and Tierney 2009), was also evidenced. The use of laughter as an anxiety inhibitor served to function as an incompatible behavior to anxiety, while video goggles were used to treat dental phobia. The goggles provided access to a video which served as a distractor from the ongoing dental exam. The current review found that the majority of the articles reported the use of multi-component interventions $(n=14$; Davis et al. 2007; Ellis et al. 2006; Gillis et al. 2009; Isong et al. 2014; Jackson and King 1982; Karnezi and Tierney 2009; Love et al. 1990; Luiselli 1978; Luscre and Center 1996; Rapp et al. 2005; Ricciardi et al. 2006; Schleismann and Gillis 2011; Schmidt et al. 2013; Shabani and Fisher 2006).

The duration of treatment was also examined across studies and reporting of this variable was infrequent. However, of the seven studies which provided this information (Gillis et al. 2009; Isong et al. 2014; Karnezi and Tierney 2009; Luiselli 1978; Rapp et al. 2005; Ricciardi et al. 2006; Schleismann and Gillis 2011), treatment duration ranged from one session (Isong et al. 2014) to 62 sessions (Gillis et al. 2009). 


\section{Settings}

The interventions were primarily conducted in school settings ( $n=6$; Chok et al. 2010; Gillis et al. 2009; Jackson and King 1982; Koegel et al. 2004; Luscre and Center 1996; Schmidt et al. 2013), in the participant's home ( $n=3$; Ellis et al. 2006; Koegel et al. 2004; Love et al. 1990), or in therapist clinics ( $n=4$; Karnezi and Tierney 2009; Koegel et al. 2004; Schleismann and Gillis 2011; Shabani and Fisher 2006), hospital ( $n=3$; Davis et al. 2007; Isong et al. 2014; Ricciardi et al. 2006). However, a number of interventions were delivered in the location central to the feared stimuli, for example, in a public swimming pool ( $n=1$; Rapp et al. 2005) or on the school bus ( $n=1$; Luiselli 1978).

\section{Outcomes}

All studies reported an overall reduction or elimination of the targeted fear or phobia. In addition to this, all twelve studies which reported the occurrence of challenging behavior in the presence of the aversive stimuli reported a subsequent decrease in, or elimination of, challenging behavior as a result of intervention (Chok et al. 2010; Davis et al. 2007; Ellis et al. 2006; Gillis et al. 2009; Jackson and King 1982; Koegel et al. 2004; Love et al. 1990; Luiselli 1978; Rapp et al. 2005; Ricciardi et al. 2006; Schmidt et al. 2013; Shabani and Fisher 2006). Thirteen studies included assessment of maintenance effects (Chok et al. 2010; Davis et al. 2007; Ellis et al. 2006; Gillis et al. 2009; Jackson and King 1982; Karnezi and Tierney 2009; Koegel et al. 2004; Love et al. 1990; Luiselli 1978; Ricciardi et al. 2006; Schleismann and Gillis 2011; Schmidt et al. 2013; Shabani and Fisher 2006), all of which reported positive outcomes over durations ranging up to several days post-intervention (Schmidt et al. 2013) to 3 years (Karnezi and Tierney 2009). Furthermore, four studies (Chok et al. 2010; Karnezi and Tierney 2009; Luscre and Center 1996; Rapp et al. 2005) reported the generalization of treatment effects. Three of these studies reported generalization of treatment effects across untreated settings (Karnezi and Tierney 2009; Luscre and Center 1996; Rapp et al. 2005), while the remaining study reported generalization across stimuli (Chok et al. 2010).

For 12 of the 15 single subject research design studies, outcomes were measured through visual analysis of the data (Chok et al. 2010; Davis et al. 2007; Ellis et al. 2006; Gillis et al. 2009; Koegel et al. 2004; Love et al. 1990; Luscre and Center 1996; Rapp et al. 2005; Ricciardi et al. 2006; Schleismann and Gillis 2011; Schmidt et al. 2013; Shabani and Fisher 2006). The majority of studies used this method to show an increase in acceptance behaviors and/or a decrease in fear behavior. In addition to this, one study (Chok et al. 2010) showed that a reduction in the behavioral exhibition of fear was reduced in line with a clear reduction in the associated physiological arousal. However, an additional study examining the physiological response to feared stimuli failed to evidence any impact of intervention on participants' physiological arousal (Isong et al. 2014). The included studies were also examined for information on any social validity assessments conducted. Only one study (Ellis et al. 2006) provided information, in the form of positive outcomes on a treatment satisfaction rating scale completed by parents, on the social validity of the intervention techniques employed.

\section{Evidence-Based Practice Evaluation}

The analysis of the extant research on treating fears and phobias among those with ASD, in accordance with the criteria outlined by Chambless and Hollon (1998), revealed that there is empirical support for a number of the interventional components evaluated by the studies reviewed. Table 2 presents a summary of the intervention techniques employed across the included studies along with, the research support for each, and the corresponding classification of their empirical support. Based on the Chambless and Hollon (1998) criteria a number of treatments including reinforcement procedures, modeling and exposure may be considered empirically supported. All additional interventional techniques were classified as promising but lacking empirical support. No interventions were identified to be ineffective.

\section{Discussion}

Previous research has suggested that fears and phobias are prevalent among children diagnosed with ASD, and emerge in response to a greater variety of stimuli than has been observed among children with other developmental disabilities, or those who are typically developing. Given such findings, the relatively small number of studies that have empirically examined treatments for such problematic responses to stimuli is somewhat surprising. However, findings of the extant research with regard to the type of fears treated, and the empirical support for the interventions used, are largely in line with the research on the treatment of fears and phobias among typically developing children (Davis and Ollendick 2005) or children with intellectual disabilities (Jennett and Hagopian 2008).

This review found evidence of interventions for some common childhood fears, such as dogs, doctors, and dentists; however, the majority of the fears were unusual in nature, including skin lotions, going outside, showering, swimming pools, animatronic objects, and riding the school bus. This corresponds with previous research which has suggested that fears and phobias emerge in response to a more diverse range of stimuli and are often idiosyncratic, among children with 
Table 2 Classification of interventions employed in the treatment of fears or phobias among those with autism spectrum disorders by level of empirical support

\begin{tabular}{|c|c|c|}
\hline $\begin{array}{l}\text { Level of } \\
\text { empirical } \\
\text { support }\end{array}$ & Treatment & Research support \\
\hline \multirow[t]{3}{*}{$\begin{array}{l}\text { Evidence- } \\
\text { based }\end{array}$} & $\begin{array}{c}\text { Reinforcement } \\
\text { procedures }\end{array}$ & $\begin{array}{l}\text { Positive outcomes reported for } 29 \text { participants reported by ten } \\
\text { studies employing single-subject research designs; positive results } \\
\text { for two additional participants reported by case studies }\end{array}$ \\
\hline & Modeling techniques & $\begin{array}{l}\text { Positive outcomes for } 25 \text { participants reported by five studies } \\
\text { employing single-subject research designs }\end{array}$ \\
\hline & Exposure techniques & $\begin{array}{l}\text { Positive outcomes reported for } 23 \text { participants by five studies } \\
\text { employing single-subject research designs; positive results for one } \\
\text { additional participant reported by a case study }\end{array}$ \\
\hline \multirow[t]{12}{*}{$\begin{array}{l}\text { Promising but lacking } \\
\text { sufficient } \\
\text { evidence }\end{array}$} & Systematic desensitization & $\begin{array}{l}\text { Positive outcomes reported for eight participants by four studies } \\
\text { employing single-subject research designs; positive results for one } \\
\text { additional participant reported by one case study }\end{array}$ \\
\hline & $\begin{array}{l}\text { Video goggles (distraction } \\
\text { procedure) }\end{array}$ & $\begin{array}{l}\text { Positive outcomes reported for } 40 \text { participants by one } \\
\text { randomized controlled trial }\end{array}$ \\
\hline & Reinforced practice & $\begin{array}{l}\text { Positive outcomes for } 18 \text { participants reported by one study } \\
\text { employing a single-subject research design; positive results for } \\
\text { two additional participants reported by two case studies }\end{array}$ \\
\hline & Parent-training & $\begin{array}{l}\text { Positive outcomes reported for three participants by two studies } \\
\text { employing single-subject research designs; positive results for one } \\
\text { additional participant reported by a case study }\end{array}$ \\
\hline & Cognitive behavior therapy & $\begin{array}{l}\text { Positive outcomes reported for one participant by one study employing } \\
\text { a single-subject research design; positive results for two additional } \\
\text { participants reported by two case studies }\end{array}$ \\
\hline & Psychoeducation & $\begin{array}{l}\text { Positive outcomes for two participants reported by one study } \\
\text { employing a single-subject research design }\end{array}$ \\
\hline & Cognitive challenges & $\begin{array}{l}\text { Positive outcomes for two participants reported by one study } \\
\text { employing a single-subject research design }\end{array}$ \\
\hline & Response blocking & $\begin{array}{l}\text { Positive outcome for one participant reported by one study } \\
\text { employing a single-subject research design }\end{array}$ \\
\hline & Stimulus fading & $\begin{array}{l}\text { Positive outcome for one participant reported by one study } \\
\text { employing a single-subject research design }\end{array}$ \\
\hline & Laughter as an anxiety inhibitor (DRI) & $\begin{array}{l}\text { Positive outcome for one participant reported by one study } \\
\text { employing a single-subject research design }\end{array}$ \\
\hline & Cognitive behavior drama & $\begin{array}{l}\text { Positive outcomes for one participant reported by a single case } \\
\text { study }\end{array}$ \\
\hline & Social story & $\begin{array}{l}\text { Positive outcomes for one participant reported by a single case } \\
\text { study }\end{array}$ \\
\hline Ineffective & - & - \\
\hline
\end{tabular}

ASD (Evans et al. 2005; Matson and Love 1990; Mayes et al. 2013).

Further, the research reviewed also demonstrates a close association between fears and phobias in ASD and challenging behavior. The majority (75\%) of the included studies reported that participants engaged in behaviors, which may be considered challenging, including self-injury, aggression, and property destruction, in the presence of the feared or phobic stimulus. This finding is in line with that of Evans and colleagues (2005) who identified a greater correlation between fears or phobias and challenging behaviors among those with ASD than among those diagnosed with Down syndrome. The engagement in challenging behavior in response to the aversive stimuli may arise from the core communication deficits associated with ASD, and the difficulty of expressing such negative emotional responses appropriately. This association between challenging behaviors and responsiveness to aversive stimuli highlights the importance of careful consideration of context and various environmental stimuli when assessing the function of challenging behavior. For many of the fears targeted in the reviewed studies, such as skin lotion, swimming pools, and 
the doctor, the aversive stimuli may be sufficiently irregularly occurring or novel that the fear or phobia underlying the elicited challenging behavior may be evident. However, for some of the more common or subtle stimuli, such as the fear of auditory stimuli, going outside, or cases of social phobia, the association between the stimulus and challenging behavior may not be as clear. In such cases, traditional functional analyses may fail to yield clear results regarding behavioral function if the aversive stimulus is absent during the experimental sessions and the establishment of a relation between the specific stimulus and challenging behavior may require the use of careful descriptive assessment. Given the prevalence of fears or phobias among children with ASD, which Turner and Romanczyk (2012) suggest may be as high as $64 \%$, the more frequent consideration of potentially fearinducing or phobic stimuli during functional assessments may be warranted.

The results of the current analysis reveal that interventional studies have focused primarily on assessing and treating the observable behavioral response to the aversive stimulus with few examining the two additional components of the fear response, cognitive, and physiological reactions (Lang 1968). This finding echoes that of previous reviews which have examined treatments for fears or phobias among children with intellectual disabilities (Jennett and Hagopian 2008) and typically developing children (Davis and Ollendick 2005). Of the 16 studies included in the current review only two measured physiological arousal (Chok et al. 2010; Isong et al. 2014) while no studies measured changes in the cognitive response due to intervention. It may be argued that in order to ensure the complete elimination of a fear or phobia, all three elements of the fear response should be measured to ensure that it is completely eradicated. While Chok and colleagues (2010) demonstrated the reduction of the physiological response in line with the reduction of the behavioral response, Isong and colleagues (2014) failed to evidence any changes in the physiological reaction to the feared stimulus although the problematic behavioral response was effectively reduced. Such findings suggest that although the overt behavioral response to the stimuli may be eliminated, the physiological fear response may persist. This suggestion is supported by the research findings of Lydon et al. (2013) who conducted a preliminary study examining the heart rate patterns associated with the challenging behavior of three youths diagnosed with ASD. For one participant, noise was a noted aversive stimulus, which had previously elicited severe self-injury. Behavioral intervention had been successful in reducing the selfinjurious behavior and teaching the participant an alternative behavioral response, ear covering, to such auditory stimuli. However, the heart rate analysis revealed an increase in physiological arousal prior to engagement in ear covering in the presence of noise suggesting that the stimulus continued to produce physiological stress. A reduction in physiological arousal following the offset of ear covering was also observed. Such findings suggest that although the behavioral response to an aversive stimulus may be managed, the physiological stress associated with the stimulus may persist in some cases. In addition to this, the cognitive aspect of the feared response may also still be in existence, even if the behaviors have been altered. The assessment of the cognitive fear response may be difficult among those with ASD, given the associated communication deficits; however, the feasibility of such assessments among individuals with high-functioning autism may warrant future investigation.

The current review revealed that the interventions applied in the treatment of fears and phobias among those with ASD are in line with those used for typically developing children or children with other developmental disabilities (Davis and Ollendick 2005; Jennett and Hagopian 2008). These included reinforced practice, systematic desensitization, modeling, exposure techniques, contingent reinforcement, distraction procedures, cognitive behavioral therapy, and response blocking. In addition to these methods, a number of less common interventional techniques, such as laughter as an anxiety inhibitor (a form of DRI), cognitive behavior drama, and social stories, were also employed in the studies reviewed. Furthermore, three studies (Love et al. 1990; Luiselli 1978; Schleismann and Gillis 2011) made use of parent training to facilitate the delivery of intervention. Parent training involved the parent acting as the therapist and employing one of the other intervention methods; for example, the parent modeling the appropriate behaviors in the presence of the eliciting stimulus. Treatment delivery in this manner may be both cost effective and otherwise beneficial as research suggests the involvement of the parent in the treatment process can result in better treatment outcomes for the child (Cobham et al. 1998; Mendlowitz et al. 1999; Reaven and Hepburn 2006).

The data extracted on treatment duration (range, 1-62 treatment sessions) across the reviewed studies is also of interest. The frequent non-reporting of treatment duration is problematic as it may preclude the replication of treatment effects. Furthermore, treatment duration also represents an important component of treatment efficacy and is often a consideration for parents and clinicians when choosing a treatment approach. Thus, it is imperative that future studies in this area present this data for examination. The large variance in treatment duration (number of sessions), among studies that reported this data is also of note and may be useful in determining research direction. Future researchers should examine factors such as participant or treatment characteristics which may contribute to increased intervention efficiency. This information may be valuable to clinicians seeking to treat fears and phobias in this population.

Our ability to draw conclusions about treatment efficacy and appropriateness from the extant research is somewhat impaired by the frequent use of multi-component treatment 
packages. Many of the included studies delivered intervention comprising a number of techniques suggested to be effective by previous reviews of the literature examining the treatment of fears and phobias (Davis and Ollendick 2005; Jennett and Hagopian 2008). In this way, it is difficult to ascertain the contribution of the individual treatment components to treatment efficacy and to determine which components are necessary or sufficient for treatment success.

All studies reviewed reported treatment success and the successful reduction of the fearful or phobic behavioral response. Of the two studies assessing participants' physiological activity, one demonstrated a decrease in physiological arousal, which corresponded with the decrease in the overt behavioral response, elicited by the feared stimulus (Chok et al. 2010) while the other (Isong et al. 2014) found no effect of the intervention on physiological arousal although it successfully altered the problematic behavioral response. Such findings suggest that further research is needed to determine the impact of interventions such as those reviewed here on physiological reactions to the feared or phobic stimuli. The outcomes of maintenance and generalization assessments, measured by 81.3 and $25 \%$ of studies, respectively, were largely positive although the quality of these assessments may be considered poor in some cases (e.g., the assessment of maintenance several days post-intervention). Only one study reviewed (Ellis et al. 2006) examined the social validity of the intervention implemented with findings suggesting the participant's parents perceived the intervention positively.

The current review sought to establish evidence-based practice in the treatment of fears and phobias among those with ASD through the application of Chambless and Hollon's (1998) criteria for ascertaining the level of empirical support for the various interventional components evaluated in the studies reviewed. This analysis was significantly limited by the pervasive usage of multi-component treatment packages for the treatment of fears and phobias among those with ASD and the difficulty of determining the active components of treatment. For this reason, the analysis and its findings must be considered preliminary and interpreted with caution. However, such an analysis was considered an important undertaking given the prevalence of fears and phobias, their frequent association with challenging behavior, and the importance of identifying evidence-based practice in this area and providing recommendations for clinicians.

The results of this preliminary analysis, presented in Table 2, suggest that, for individuals diagnosed with ASD, the use of reinforcement procedures, modeling procedures, and exposure techniques during the treatment of fears or phobias appear to constitute evidence-based practice and should be utilized in the treatment of fears and phobias among persons with ASD. The current review suggests that other interventional techniques including systematic desensitization, cognitive behavior therapy and reinforced practice are likely effective but require further research. The techniques identified as evidence-based practice in the current review mirror those identified by Davis and Ollendick (2005) and Jennett and Hagopian (2008) in their examinations of evidence-based practice for the treatment of fears and phobias among typically developing children and children with intellectual disabilities respectively. While the current analysis was limited by the usage of these interventional techniques within multicomponent treatment packages, its findings with regards the various treatment components do allow for the conclusion that behavioral intervention for fears and phobias among those with ASD may be considered evidence-based practice.

The current review may be criticized for the reliance upon the treatment descriptions and classifications provided by the authors of the studies reviewed. We did not interpret or reclassify the treatments described, and reported them in the manner in which they were described. This approach was considered necessary because the operational definitions or descriptions of treatment delivered across the studies reviewed were limited. As a result, there may be a significant overlap between a number of the interventional techniques described. For example, exposure techniques, cognitive challenges, and psychoeducation may be considered elements of Cognitive Behavior Therapy rather than being viewed as individual treatment components. However, examination of such interventional components may provide a greater understanding of the actual treatment delivered and may facilitate future research which seeks to maximize the efficiency and efficacy of interventions in this area.

While the review undertaken indicates that there are a number of effective interventional techniques suitable for the treatment of fears and phobias among those with ASD, it also suggests a number of areas in which further research is required. Given the frequent employment of multicomponent treatment packages (94\% of studies), future research should endeavor to identify the effective or active elements of such treatment packages, through component analyses or other suitable experimental designs, in order to establish evidence-based practice in this area and to aid clinicians in applying the most efficient and effective treatment for fears or phobias with which children with ASD may present. The utilization of randomized controlled trials, employed by only one study in the current review, may also be valuable in furthering our knowledge in this area and promoting evidence-based practice. While the current review suggests that a number of evidence-based techniques exist for treating such problems, additional research studies should endeavor to examine these interventions within randomized controlled experimental designs to examine the comparative efficacy of the various techniques which have been suggested to be empirically supported and to establish the efficacy of those techniques deemed promising but lacking sufficient evidence. 
Furthermore, as the majority of the current research focuses solely on assessing and treating the behavioral element of fearful and phobic reactions, there exists a notable deficit in the research regarding the assessment and treatment of cognitive and physiological responses to fears and phobias in this population. The measurement of the physiological component of the fear response may be particularly useful among persons with ASD given the noted difficulties of assessing fears and phobias in this population (e.g., Grondhuis and Aman 2012; Reaven 2009; Tsai 2006) and may offer researchers a greater insight into a client's experience and response to intervention. While it is possible that interventions that target the behavioral component of the fear response lead to corresponding decrements in the cognitive and physiological aspects of the fear response, research is required to confirm or disconfirm this suggestion. Finally, of the 16 studies reviewed, only three included individuals with co-occurring intellectual disabilities. Given that the prevalence of co-occurring intellectual disabilities among those with ASD has been estimated to be $70 \%$ (La Malfa et al. 2004; Matson and Shoemaker 2009), the current body of research may under represent lowerfunctioning individuals diagnosed with ASD whose treatment needs and complexity of presentation may differ significantly from those higher-functioning individuals with ASD. Thus, future research should examine the efficacy of interventions for fears and phobias among those diagnosed with ASD and co-occurring intellectual disabilities.

\section{References}

American Psychiatric Association. (2013). Diagnostic and statistical manual of mental disorders (5th ed.). Arlington: American Psychiatric Publishing.

Beesdo, K., Knappe, S., \& Pine, D. S. (2009). Anxiety and anxiety disorders in children and adolescents: developmental issues and implications for DSM-V. The Psychiatric Clinics of North America, 32, 483-524.

Chambless, D. L., \& Hollon, S. D. (1998). Defining empirically supported therapies. Journal of Consulting and Clinical Psychology, 66, 7-18.

Chok, J. T., Demanche, J., Kennedy, A., \& Studer, L. (2010). Utilizing physiological measures to facilitate phobia treatment with individuals with autism and intellectual disability: a case study. Behavioral Interventions, 25, 325-337.

Cobham, S. N., Dadds, M. R., \& Spence, S. H. (1998). The role of parent anxiety in the treatment of childhood anxiety. Journal of the American Academy of Child and Adolescent Psychiatry, 43, 930959.

Davis, T. E., Kurtz, P. F., Gardner, A. W., \& Carman, N. B. (2007). Cognitive-behavioral treatment for specific phobias with a child demonstrating severe problem behavior and developmental delays. Research in Developmental Disabilities, 28, 546-558.

Davis, T. E., \& Ollendick, T. H. (2005). Empirically supported treatments for specific phobia in children: do efficacious treatments address the components of a phobic response? Clinical Psychology: Science and Practice, 12, 144-160.
Ellis, E. M., Ala'i-Rosales, S. S., Glenn, S. S., Rosales-Ruiz, J., \& Greenspoon, J. (2006). The effects of graduates exposure, modelling, and contingent social attention on tolerance to skin care products with two children with autism. Research in Developmental Disabilities, 27, 585-598.

Emerson, E. (2001). Challenging behaviour: analysis and intervention in people with severe intellectual disabilities. Cambridge: Cambridge University Press.

Evans, D. W., Canavera, K., Lee-Kleinpeter, F., Maccubbin, E., \& Taga, K. (2005). The fears, phobias and anxieties of children with autism spectrum disorders and down syndrome: comparisons with developmentally and chronologically age matched children. Child Psychiatry and Human Development, 36, 3-26.

Gillis, J. M., Hammond Natof, T., Lockshin, S. B., \& Romancczyk, R. G. (2009). Fear of routine physical exams in children with autism spectrum disorders: prevalence and intervention effectiveness. Focus on Autism and Other Developmental Disabilities, 24, 156-168.

Grondhuis, S. N., \& Aman, M. G. (2012). Assessment of anxiety in children and adolescents with autism spectrum disorders. Research in Autism Spectrum Disorders, 6, 1345-1365.

Isong, I. A., Rao, S. R., Holifield, C., Iannuzzi, D., Hanson, E., Ware, J., \& Nelson, L. P. (2014). Addressing dental fear in children with autism spectrum disorders: a randomized controlled pilot study using electronic screen media. Clinical Pediatrics, 53, 230-237.

Jackson, H. J., \& King, N. J. (1982). The therapeutic management of an autistic child's phobia using laughter as the anxiety inhibitor. Behavioral Psychotherapy, 10, 364-369.

Jennett, H. K., \& Hagopian, L. P. (2008). Identifying empirically supported treatments for phobic avoidance in individuals with intellectual disabilities. Behavior Therapy, 39, 151-161.

Karnezi, H., \& Tierney, K. (2009). A novel intervention to address fears in children with Asperger syndrome: a pilot study of the Cognitive Behavior Drama (CBD) model. Behavior Change, 26, 271-282.

Koegel, R. L., Openden, D., \& Koegel, L. K. (2004). A systematic desensitazation paradigm to treat hypersensitivity to auditory stimuli in children with autism in family contexts. Research \& Practice for Persons with Severe Disabilities, 29, 122-134.

La Malfa, G., Lassi, S., Bertelli, M., Salvini, R., \& Placidi, G. F. (2004) Autism and intellectual disability: a study of prevalence on a sample of the Italian population. Journal of Intellectual Disability Research, 48(3), 262-267.

Lang, P. J. (1968). Fear reduction and fear behavior: problems in treating a construct. In J. M. Shlien (Ed.), Research in psychotherapy (pp. 90-103). Washington: American Psychological Association.

LeBeau, R. T., Glenn, D., Liao, B., Wittchen, H. U., Beesdo-Baum, K., Ollendick, T., \& Craske, M. G. (2010). Specific phobia: a review of DSM-IV specific phobia and preliminary recommendations for DSM-V. Depression and Anxiety, 27, 148-167.

Leyfer, O. T., Folstein, S. E., Bacalman, S., Davis, N. O., Dinh, E., Morgan, J., \& Lainhart, J. E. (2006). Comorbid psychiatric disorders in children with autism: interview development and rates of disorders. Journal of Autism \& Developmental Disorders, 36, 849-861.

Lichtenstein, P., \& Annas, P. (2000). Heritability and prevalence of specific fears and phobias in childhood. Journal of Child Psychology and Psychiatry, 927-937.

Love, S. R., Matson, J. L., \& West, D. (1990). Mothers as effective therapists for autistic children's phobias. Journal of Applied Behavior Analysis, 23, 379-385.

Luiselli, J. K. (1978). Treatment of an autistic child's fear of riding a school bus through exposure and reinforcement. Journal of Behavior Therapy and Experimental Psychiatry, 9, 169-172.

Luscre, D. M., \& Center, D. B. (1996). Procedures for reducing dental fear in children with autism. Journal of Autism and Developmental Disorders, 26, 547-556.

Lydon, S., Healy, O., \& Dwyer, M. (2013). An examination of heart rate during challenging behavior in autism spectrum disorder. 
Journal of Developmental and Physical Disabilities, 25(1), 149170.

Marks, I. M. (1987). Fears, phobias, and rituals. New York: Oxford University Press.

Matson, J. L., \& Love, S. R. (1990). A comparison of parent-reported fear for autistic and non-handicapped age-matched children and youth. Australian and New Zealand Journal of Developmental Disabilities, 16, 349-357.

Matson, J. M., \& Shoemaker, M. (2009). Intellectual disability and its relationship to autism spectrum disorders. Research in Developmental Disabilities, 30, 1107-1114.

Mayes, S. D., Calhoun, S. L., Aggarwal, R., Baker, C., Mathapati, S., Molitoris, S., \& Mayes, R. D. (2013). Unusual fears in children with autism. Research in Autism Spectrum Disorders, 7, 151-158.

Mayes, S. D., Calhoun, S. L., Mayes, R. D., \& Molitoris, S. (2012). Autism and ADHD: overlapping and discriminating symptoms. Research in Autism Spectrum Disorders, 6, 277-285.

Mendlowitz, S. L., Manassis, K., Bradley, S., Scapillato, D., Miezitis, S., \& Shaw, B. F. (1999). Cognitive-behavioral group treatments in childhood anxiety disorders: the role of parent involvement. Journal of the American Academy of Child and Adolescent Psychiatry, 38, 1223-1229.

Muris, P., Merckelbach, H., Mayer, B., \& Prins, E. (2000). How serious are common childhood fears? Behaviour Research and Therapy, 38 , $217-228$

Muris, P., Steerneman, P., Merckelback, H., Holdrinet, I., \& Meesters, C. (1998). Comorbid anxiety symptoms in children with pervasive developmental disorders. Journal of Anxiety Disorders, 12, 387-393.

Nesse, R. M., Curtis, G. C., Thyer, B. A., McCann, D. S., Huber-Smith, M. J., \& Knopf, R. F. (1985). Endocrine and cardiovascular responses during phobic anxiety. Psychosomatic Medicine, 47, 320-332.

Ollendick, T. H., \& King, N. J. (1998). Empirically supported treatments for children with phobic and anxiety disorders: current status. Journal of Clinical Child Psychology, 27, 156-167.

Ollendick, T. H., King, N. J., \& Muris, P. (2002). Fears and phobias in children: phenomenology, epidemiology and aetiology. Child and Adolescent Mental Health, 7, 98-106.

Rapp, J. T., Vollmer, T. R., \& Hovanetz, A. N. (2005). Evaluation and treatment of swimming pool avoidance exhibited by an adolescent girl with autism. Behavior Therapy, 36, 101-105.

Reaven, J. A. (2009). Children with high-functioning autism spectrum disorders and co-occurring anxiety symptoms: implications for assessment and treatment. Journal for Specialists in Pediatric Nursing, 14, 192-199.

Reaven, J., \& Hepburn, S. (2006). The parent's role in the treatment of anxiety symptoms in children with high-functioning autism spectrum disorders. Mental Health Aspects of Developmental Disabilities, 9, 73-80.

Rieffe, C., Meerum Terwogt, M., \& Kotronopoulou, K. (2007). Awareness of single and multiple emotions in high functioning children with autism. Journal of Autism and Developmental Disorders, 37, 455-465.

Ricciardi, J. N., Luiselli, J. K., \& Camare, M. (2006). Shaping approach responses as intervention for specific phobia in child with autism. Journal of Applied Behavior Analysis, 39, 445-448.

Schleismann, K. D., \& Gillis, J. M. (2011). The treatment of social phobia in a young boy with Asperger's disorder. Cognitive and Behavioral Practice, 18, 515-529.

Schmidt, J. D., Luiselli, J. K., Rue, H., \& Whalley, K. (2013). Graduated exposure and positive reinforcement to overcome setting and activity avoidance in an adolescent with autism. Behavior Modification, $37,128-142$.

Shabani, D. B., \& Fisher, W. W. (2006). Stimulus fading and differential reinforcement for the treatment of needle phobia in a youth with autism. Journal of Applied Behavior Analysis, 39, 449-452.

Tsai, L. (2006). Diagnosis and treatment of anxiety disorders in individuals with autism spectrum disorder. In M. G. Baron, J. Groden, G. Groden, \& L. P. Lipsitt (Eds.), Stress and coping in autism. New York: Oxford University Press.

Turner, L. B., \& Romanczyk, R. G. (2012). Assessment of fear in children with autism spectrum disorder. Research in Autism Spectrum Disorders, 6, 1203-1210.

Van Houtem, C. M. H. H., Laine, M. L., Boomsma, D. I., Ligthart, L., van Wijk, A. J., \& de Jongh, A. (2013). A review and meta-analysis of the heritability of specific phobia subtypes and corresponding fears. Journal of Anxiety Disorders, 27, 379-388.

Van Steensel, F. J. A., Bogels, S. M., \& Perrin, S. (2011). Anxiety disorders in children and adolescents with autistic spectrum disorders: a meta-analysis. Clinical Child \& Family Psychology Review, $14,302-317$.

White, S. W., Schry, A. R., \& Maddox, B. B. (2012). Brief report: the assessment of anxiety in high-functioning adolescents with autism spectrum disorder. Journal of Autism and Developmental Disorders, $42,1138-1145$. 